\title{
Retraction Note to: Gene expression profiling analysis of locus coeruleus in idiopathic Parkinson's disease by bioinformatics
}

\author{
Shuqin Cui ${ }^{1} \cdot$ Hanwen Sun ${ }^{1,2} \cdot X^{\text {Xiangling }} \mathrm{Gu}^{1} \cdot \mathrm{E} \mathrm{Lv}^{3} \cdot$ Yancong Zhang $^{1} \cdot$ \\ Pingxuan Dong ${ }^{1} \cdot$ Chunhua $\mathrm{Fu}^{1} \cdot$ Chao $\mathrm{Zhu}^{1}$
}

Published online: 17 August 2015

(C) Springer-Verlag Italia 2015

\section{Retraction to: Neurol Sci (2015) 36:97-102 \\ DOI 10.1007/s10072-014-1889-z}

The Publisher and Editor retract this article in accordance with the recommendations of the Committee on Publica tion Ethics (COPE). After a thorough investigation we have strong reason to believe that the peer review process was compromised.

The online version of the original article can be found under doi:10.1007/s10072-014-1889-z.

\section{Hanwen Sun}

hanwensun001@163.com

1 China Shandong Provincial Engineering Laboratory of New Pharmaceutical Excipients, Sustained and Controlled Release Technology, College of Medicine and Nursing, Dezhou University, Dezhou city 253023, Shandong, China

2 Department of medicine, Dezhou University, No. 566, West Daxue Road, Decheng District, Dezhou city 253023, Shandong, China

3 Department of Neurobiology, Capital Medical University, Beijing 100069, China 